\title{
Prognostic impact of sarcopenia on immune-related adverse events in malignancies received immune checkpoint inhibitors: a systematic review and meta-analysis
}

\author{
Shuluan Li ${ }^{1 \#}$, Tianyu Wang ${ }^{2 \#}$, Wenjuan Lai ${ }^{3 \#}$, Mingying Zhang ${ }^{4,5}$, Boran Cheng ${ }^{4}$, Shubin Wang ${ }^{4}$, \\ Gangling Tong ${ }^{4}$ \\ ${ }^{1}$ Department of Medical Oncology, National Cancer Center/National Clinical Research Center for Cancer/Cancer Hospital \& Shenzhen Hospital, \\ Chinese Academy of Medical Sciences and Peking Union Medical College, Shenzhen, China; ${ }^{2}$ Department of Breast Surgery, National Cancer \\ Center/National Clinical Research Center for Cancer/Cancer Hospital \& Shenzhen Hospital, Chinese Academy of Medical Sciences and Peking \\ Union Medical College, Shenzhen, China; ${ }^{3}$ Nursing Department, Peking University Shenzhen Hospital, Shenzhen, China; ${ }^{4}$ Department of \\ Oncology, Peking University Shenzhen Hospital, Shenzhen Key Laboratory of Gastrointestinal Cancer Translational Research, Cancer Institute of \\ Shenzhen-PKU-HKUST Medical Center, Shenzhen, China; ${ }^{5}$ Shantou University Medical College, Shantou, China \\ Contributions: (I) Conception and design: S Wang, G Tong; (II) Administrative support: S Wang, G Tong; (III) Provision of study materials or \\ patients: S Wang, G Tong; (IV) Collection and assembly of data: S Li, T Wang, W Lai, M Zhang, B Cheng; (V) Data analysis and interpretation: \\ S Li, T Wang, W Lai, M Zhang, B Cheng; (VI) Manuscript writing: All authors; (VII) Final approval of manuscript: All authors. \\ \#These authors contributed equally to this work. \\ Correspondence to: Dr. Shubin Wang; Dr. Gangling Tong. Department of Oncology, Peking University Shenzhen Hospital, Shenzhen Key Laboratory \\ of Gastrointestinal Cancer Translational Research, Cancer Institute of Shenzhen-PKU-HKUST Medical Center, Shenzhen 518036, China. \\ Email: wangshubin2013@163.com; tg1221747@sohu.com.
}

Background: Whether sarcopenia has an impact on immune-related adverse events (irAEs) in patients with malignant neoplasms receiving immune checkpoint inhibitors (ICIs) is not consistent. This study aimed to evaluate the impact of sarcopenia on all grades of irAEs.

Methods: PubMed, Embase, and Cochrane Library databases were systematically searched for related studies up to May 2021. Eligible studies were included according to the PICOS criteria. The risk of bias of the included studies was assessed according to the Newcastle-Ottawa Scale (NOS). The odds ratio (OR), corresponding to the $95 \%$ confidence interval (CI) of all grades of irAEs, was collected and analyzed, and a further subgroup analysis of serious adverse events was conducted. All analyses were conducted using the RevMan 5.4 software downloaded from the Cochrane website. The heterogeneity and sensitivity of the study were assessed.

Results: Of the 135 references identified, only 8 studies were analyzed, including 519 patients comprising 250 with sarcopenia and 269 without sarcopenia. No obvious bias was observed in the included studies. An increased incidence of irAEs was not observed in patients with sarcopenia at pre-immunotherapy compared to those without sarcopenia. The OR and corresponding 95\% CI were 0.97 and $0.62-1.53$, respectively $(\mathrm{P}=0.90)$, with low heterogeneity $\left(\mathrm{P}=0.17, \mathrm{I}^{2}=32 \%\right)$. Further, severe adverse events were analyzed in three studies, and the results showed that sarcopenia was not related to irAEs $(\mathrm{P}=0.97)$.

Conclusions: Malignancies with sarcopenia at pre-immunotherapy may not increase the incidence of irAEs, and sarcopenia may not be a predictive factor for irAEs.

Keywords: Colorectal cancer; sarcopenia; immune checkpoint inhibitors (ICIs); immune-related adverse events (irAEs)

Submitted Jul 31, 2021. Accepted for publication Nov 12, 2021.

doi: $10.21037 /$ tcr-21-1470

View this article at: https://dx.doi.org/10.21037/tcr-21-1470 


\section{Introduction}

Immune checkpoint inhibitors (ICIs), as an antitumor therapy, are currently widely used in patients with malignant tumors because of their therapeutic effects, especially in those patients with advanced stages and weaker physiques who cannot tolerate chemotherapy. Immunotherapy has been shown to improve long-term survival while reducing treatment adverse events compared to that of conventional chemotherapy $(1,2)$. Although the overall tolerance for ICIs is better than that for chemotherapy, they may still induce immune-related adverse events (irAEs) and even severe adverse events. Some factors may predict the risk of adverse events. Different infusion times may affect irAEs, and the incidence of irAEs of any grade was higher in a 30-min infusion group than that in a 60 -min infusion group (3). Different ICIs induce different irAEs. irAEs were more frequent in patients receiving programmed cell death protein 1 (PD-1) inhibitors than those receiving programmed death-ligand 1 (PD-L1) inhibitors (4). However, there is still no gold standard or consensus to assess this possibility.

Sarcopenia is characterized by loss of skeletal muscle mass and function, which most commonly occurs in patients with cancer, especially in metastatic or advanced cases. Several studies have demonstrated that sarcopenia is associated with poor outcomes in patients undergoing chemotherapy, surgery, or immunotherapy (5-8) and increased the cytotoxicity of chemotherapy drugs and poor quality of life $(9,10)$; however, the adverse events induced by ICIs are still inconsistent. Sarcopenic obesity was related to earlier irAEs in patients with melanoma (11). However, our team's early meta-analysis showed that sarcopenia was not associated with irAEs (12). Considering the few included articles, this is the first meta-analysis to evaluate only the influence of sarcopenia on irAEs. We present the following article in accordance with the PRISMA reporting checklist (available at https://dx.doi.org/10.21037/tcr-21-1470).

\section{Methods}

\section{Data source and search strategy}

The literature was systematically searched by two reviewers (Shuluan Li and Tianyu Wang) for related studies in PubMed, Embase, and the Cochrane Library from inception until May 2021, using the following search terms: "neoplasia", "neoplasias", "neoplasm", "tumors", "tumor", "cancer”, "cancers", "malignancy", "malignancies", "malignant neoplasms", "malignant Neoplasm", "neoplasm malignant", "neoplasms malignant", "benign neoplasms", "neoplasms, benign", "benign neoplasm", "Neoplasm, Benign", "checkpoint Inhibitors, Immune", "immune Checkpoint Inhibitor", "checkpoint Inhibitor, Immune", "immune checkpoint blockers", "Checkpoint Blockers, Immune", "Immune Checkpoint Blockade", "Checkpoint Blockade, Immune", "Immune Checkpoint Inhibition", "Checkpoint Inhibition, Immune", "PD-L1 Inhibitors", "PD L1 Inhibitors", "PD-L1 Inhibitor", "PD L1 Inhibitor", "Programmed Death-Ligand 1 Inhibitors", "Programmed Death Ligand 1 Inhibitors", "CTLA-4 Inhibitors", "CTLA 4 Inhibitors", "CTLA-4 Inhibitor", "CTLA 4 Inhibitor", "T-Lymphocyte-Associated Protein 4 Inhibitors", "Cytotoxic T Lymphocyte Associated Protein 4 Inhibitors", "Cytotoxic T-Lymphocyte-Associated Protein 4 Inhibitor", "Cytotoxic T Lymphocyte Associated Protein 4 Inhibitor", "PD-1 Inhibitors", "PD 1 Inhibitors", "PD-1 Inhibitor", "Inhibitor, PD-1", "Programmed Cell Death Protein 1 Inhibitor", "Programmed Cell Death Protein 1 Inhibitors", "PD-1-PD-L1 Blockade", "Blockade, PD-1-PD-L1", "PD-1-PD-L1 Blockade", "Sarcopenia", "Myopenia", "Muscle atrophy", "Muscle wasting", "Muscle attenuation", "Muscle deletion", "Muscle loss", "Muscle weak", "Muscle reduction", "Muscle strength", "Muscle mass", "Muscular atrophy", "Muscular wasting”, "Muscular attenuation", "Muscular deletion”, "Muscular loss", "Muscular weak", "Muscular reduction", "Muscular strength", "Muscular mass", "Drug Related Side Effects and Adverse Reactions", "Side Effects of Drugs", "Drug Side Effects", "Drug Side Effect", "Effects, Drug Side", "Side Effect, Drug", "Side Effects, Drug", "Adverse Drug Reaction", "Adverse Drug Reactions", "Drug Reaction, Adverse", "Drug Reactions, Adverse", "Reactions, Adverse Drug", "Adverse Drug Event”, “Adverse Drug Events”, "Drug Event, Adverse”, "Drug Events, Adverse", "Drug Toxicity", “Toxicity, Drug”, "Drug Toxicities" and "toxicities, Drug". Moreover, the collected reference lists were screened to identify the relevant studies.

\section{Inclusion criteria}

The following were the inclusion criteria: (I) patients: patients diagnosed with malignant tumors; (II) intervention methods: immunotherapy as the main method, which included PD-1 or PD-L1 inhibitors or cytotoxic T lymphocyte-associated protein 4 (CTLA-4) inhibitors; (III) comparison factor: sarcopenia compared with those without 
sarcopenia, assessed by computer tomography (CT) imaging before immunotherapy; (IV) outcome: number of patients with irAEs; and (V) study design: randomized controlled clinical trials and observational research. All studies should be full texts of publications. Sarcopenia was defined as skeletal muscle index (SMI) or psoas muscle index (PMI) at the level of the third lumbar vertebra (L3), having a definite cutoff value.

\section{Exclusion criteria}

The exclusion criteria were as follows: (I) the patients did not have malignant tumors and did not receive ICI immunotherapy; (II) the method of diagnosing sarcopenia was not CT imaging; (III) the study did not have a control group (patients without sarcopenia); and (IV) the study was a review, case report, letter, comment, or meta-analysis.

\section{Data extraction and quality assessment}

Two authors (Shuluan Li and Tianyu Wang) independently screened the identified titles and abstracts to determine the related studies. If an abstract had potential, the full text was obtained. If an article was excluded, two authors had to agree. The data collected were as follows: first author, publication country, year of publication, sample size, ICI drug, sarcopenia evaluation method and cutoff value, irAEs of any grade, and severe irAEs. The quality of included studies was assessed according to the Newcastle-Ottawa Scale (NOS) criteria. Discrepancies were addressed for literature search, data extraction, and risk of bias assessment through discussions by the two authors.

\section{Statistical analysis}

The impact of pre-immunotherapy sarcopenia on irAEs was evaluated using the odds ratio (OR) and the corresponding $95 \%$ confidence interval (CI). The heterogeneity of the included studies was determined using $\mathrm{I}^{2}$ statistics and the Cochran's $\mathrm{Q}$ test. When $\mathrm{I}^{2} \geq 50 \%$ and/or $\mathrm{P}$ for the $\mathrm{Q}$ test $\leq 0.10$, this was regarded to have significant heterogeneity. Otherwise, if a fixed-effects analysis was performed, a random-effects analysis was also conducted. Sensitivity analysis was performed using the leave-one-out method (sequentially removing one study each time). Publication bias was assessed using a funnel plot. All analyses were performed using the RevMan 5.4 software. Statistical significance was defined as a two-sided $\mathrm{P}<0.05$.

\section{Results}

\section{Study selection and characteristics}

A total of 135 references were identified, and 35 were excluded because of duplicative titles. In addition, 90 references were excluded due to no obvious correlation with the inclusion criteria. Ten references were eligible, and the full texts were read carefully. One review and one study that did not include the number of patients with irAEs were identified and excluded. Finally, only eight eligible studies were included in the meta-analysis (Figure 1).

These eight studies were all retrospective in nature, including 519 patients (250 with sarcopenia and 269 without sarcopenia with lung cancer, melanoma, renal cell carcinoma, urothelial carcinoma, gastrointestinal cancer, soft tissue sarcoma, prostate adenocarcinoma, cervical cancer, and others). Six studies evaluated irAEs of any grade, and three studies reported serious irAEs. The main characteristics of the included studies are shown in Table 1 (8,11,13-18).

\section{Assessment of quality and risk of bias in included studies}

Randomized controlled trials in the three databases were not retrieved; therefore, the nine-point NOS was used to evaluate the quality assessment and risk of bias of the analysis of the included studies. The eight articles included had no obvious bias according to the NOS criteria. The evaluation results are shown in Table 1.

\section{Impact of sarcopenia on irAEs}

All studies reported irAEs, six articles reported irAEs of any grade, three documented severe adverse events, and three described various clinical manifestations of irAEs. Our meta-analysis showed that no difference was observed in the irAEs between patients with and without sarcopenia $(\mathrm{P}=0.90)$. The OR and corresponding 95\% CI were 0.97 (0.62-1.53) with low heterogeneity $\left(\mathrm{P}=0.17, \mathrm{I}^{2}=32 \%\right)$ using a fixed-effects model, and then a random-effects model analysis was performed, with the same results (Figure 2).

We further analyzed the severe adverse events, including three studies, and the results showed that sarcopenia was not related to irAEs $(\mathrm{P}=0.97)$, with an $\mathrm{OR}$ and corresponding 95\% CI of 1.02 and $0.41-2.52$, with moderate heterogeneity $\left(\mathrm{P}=0.12, \mathrm{I}^{2}=54 \%\right)$ (Figure 2). Finally, we further explored whether obesity in patients with sarcopenia had an impact on irAEs in the included studies. Heidelberger et al. (11) reported that lean body mass and muscle loss were not 


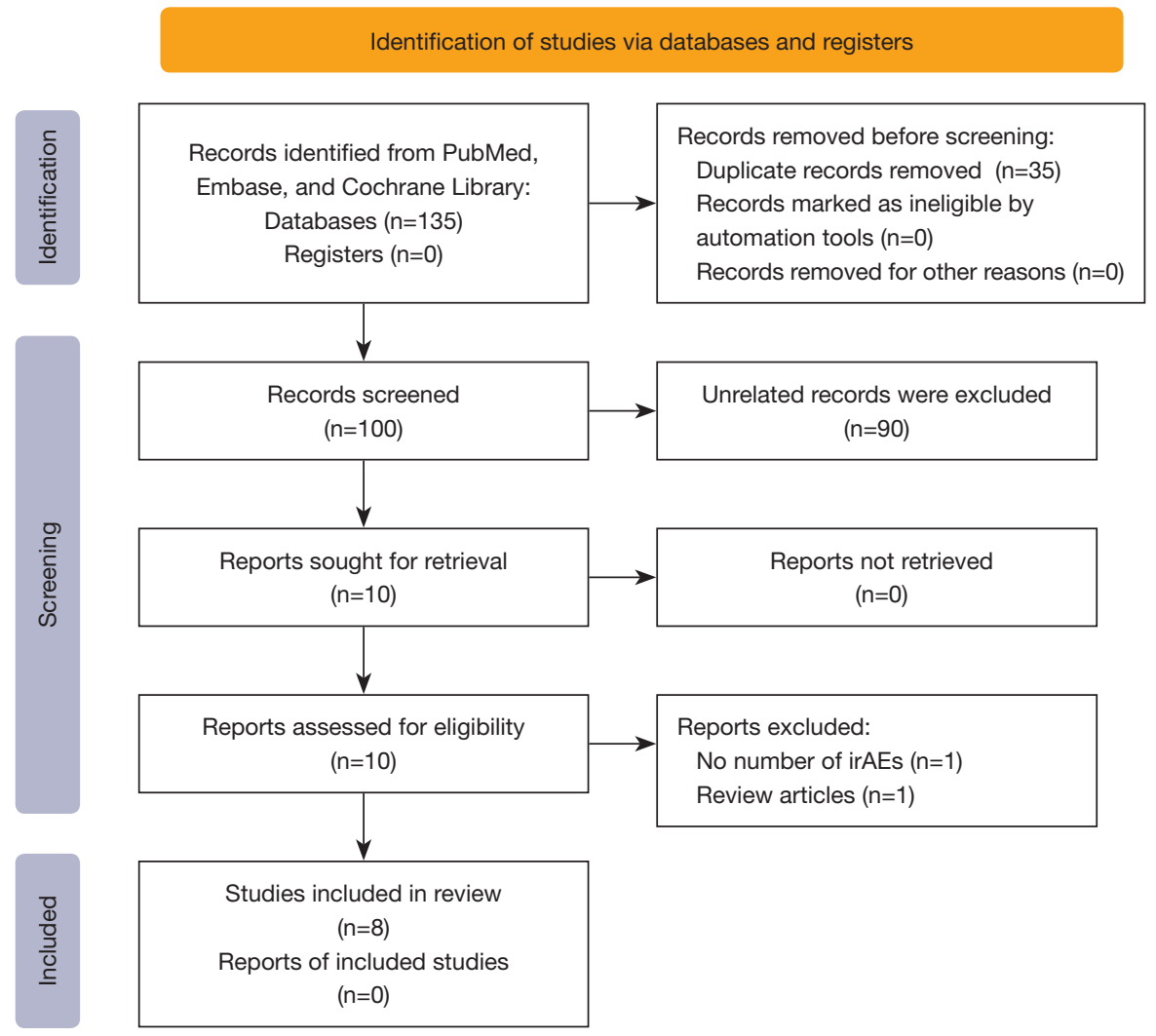

Figure 1 A flowchart of the study selection process. irAEs, immune-related adverse events.

associated with increased toxicity. However, patients with increased body mass index (BMI) experienced more toxicity, and the correlation was even stronger among female patients with $\mathrm{BMI} \geq 25 \mathrm{~kg} / \mathrm{m}^{2}$ (overweight) and sarcopenia (muscle loss) $(\mathrm{P}=0.01)$. Hirsch et al. (16) reported that patients with sarcopenia had more irAEs than those without sarcopenia $(\mathrm{OR}=3.86 ; 95 \% \mathrm{CI}: 1.40-13.1 ; \mathrm{P}=0.03)$, which was an independent prognostic factor for irAEs. However, obesity in patients with sarcopenia was not significantly related to the incidence of irAEs.

\section{Sensitivity analysis and publication bias}

Sensitivity analysis was performed by sequentially leaving one study out and repooling the remaining studies, and similar results were obtained. Publication bias of irAEs, using a funnel plot, was not observed (Figure 3).

\section{Discussion}

Sarcopenia is a component of frailty syndrome (19).
Sarcopenia is characterized by muscle loss or atrophy, which is related to ageing, reduced physical activity, and chronic diseases (20). Current research has put forward a variety of theories explaining the mechanism of muscle changes in sarcopenia, including changes in satellite cell recruitment, anabolic signals, protein oxidation, inflammation, and developmental factors $(21,22)$. Sarcopenia has widely been recognized in the field of oncology. Sarcopenia can have a negative impact on tumor treatment and can even cause falls, fractures, and disabilities, which can lead to a decline in the quality of life of patients with tumors and increase the cost of medical care (23).

Cancer immunotherapy treats cancer by artificially stimulating the immune system and improving the natural resistance of the immune system to cancer (24). In recent years, the success of several immunotherapies, such as the use of the anti-CTLA-4, antibody ipilimumab and the antiPD-1 antibody in 2011 and 2014 (25), respectively, for the treatment of melanoma has been documented. Tumors can use these checkpoints to protect themselves from the immune system (26). Blocking these negative feedback 


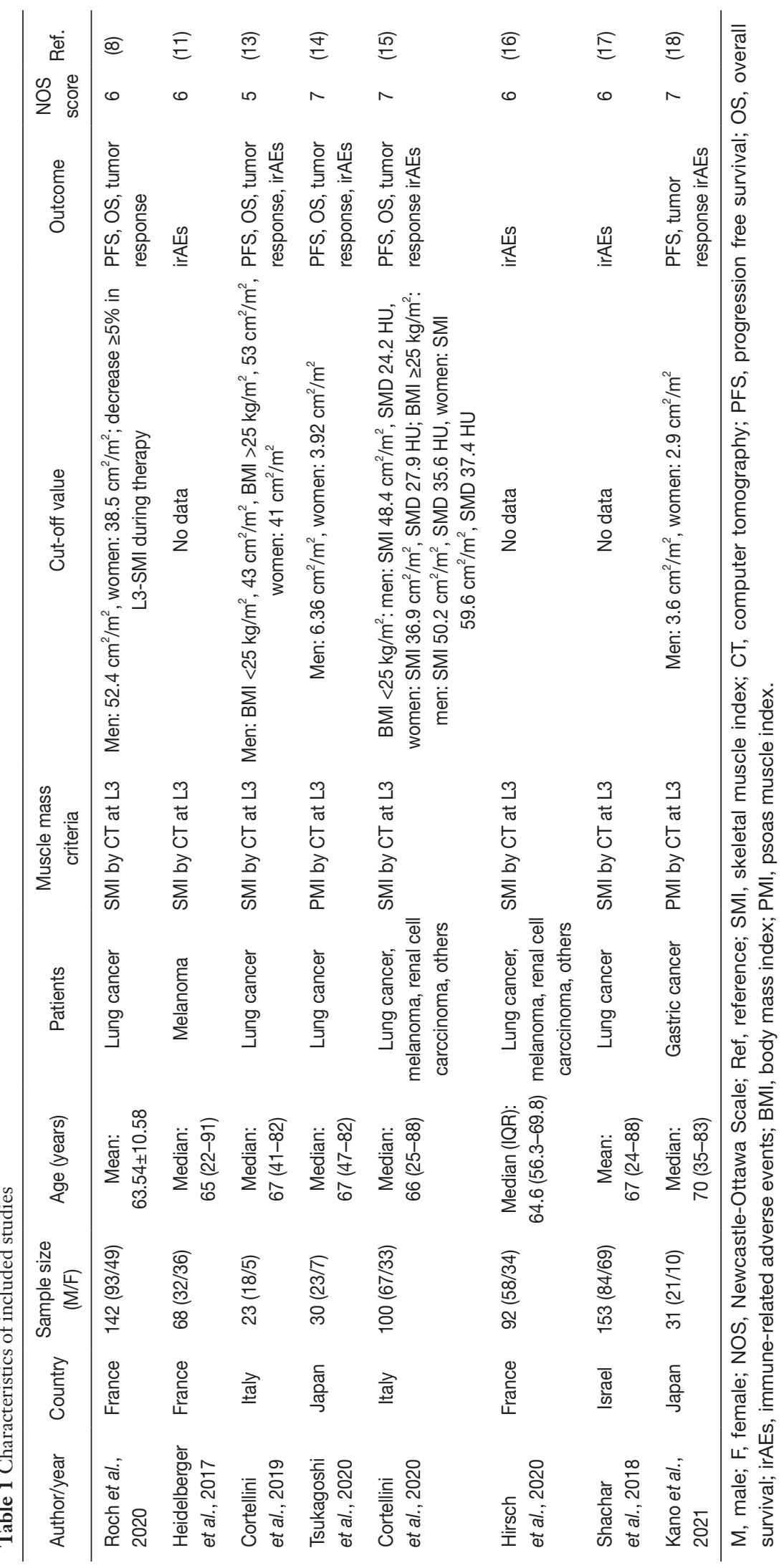




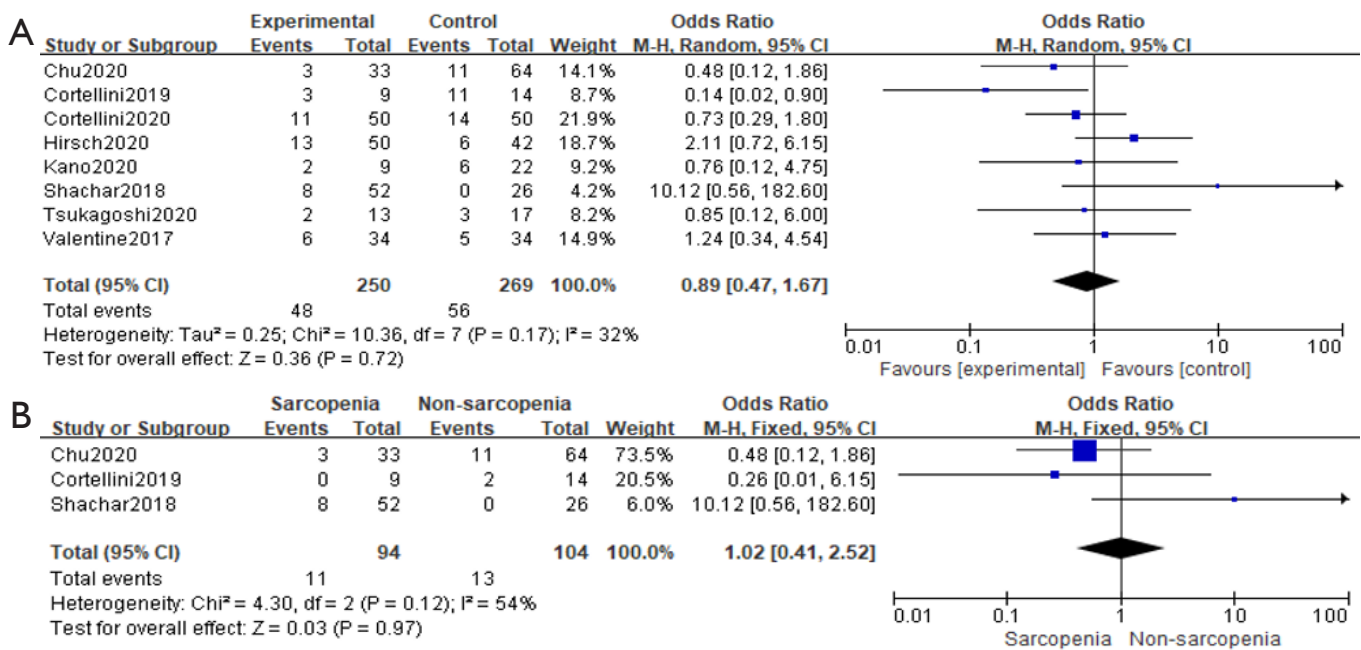

Figure 2 Forest plot of the impact of sarcopenia on irAEs. (A) Patients with sarcopenia with irAEs of any grade and (B) patients with sarcopenia with severe irAEs. CI, confidence interval; irAEs, immune-related adverse events.

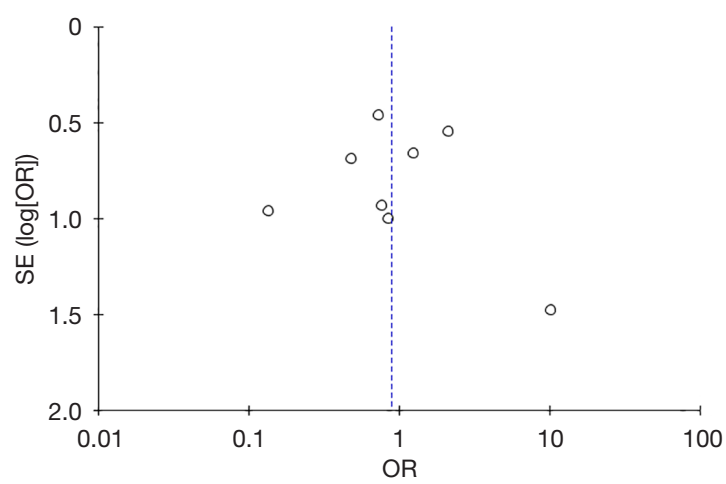

Figure 3 Funnel plot of the impact of publication bias. SE, standard error; OR, odds ratio.

signals in immune cells leads to an enhanced immune response to the tumor (27). However, patients receiving checkpoint blockers (especially the anti-CTLA-4 antibody) or a combination of checkpoint-blocking antibodies are at high risk of irAEs affecting the skin, gastrointestinal, endocrine, or liver (28). This may be due to the induction of a large number of $\mathrm{T}$ cells through injection of checkpoint blockers into the bloodstream (29). In general, immunotherapy is aimed at cancer patients as a whole, with off-target effects and common toxicity (30).

It is now clear that sarcopenia affects the toxicity of chemotherapy drugs in cancer patients (31), increases the complications of surgical treatment (32), and is related to the poor quality of life in patients with cancer (33). At the same time, various studies showed that the presence of sarcopenia was poorly correlated with the efficacy of immunotherapy, not only in lung cancer, but also in melanoma, renal cell carcinoma, urothelial carcinoma, and others (7,34-38). How muscle loss affects irAEs in patients with cancer receiving immunotherapy is still unclear. Some scholars believe that the increase in adverse reactions may be induced by immunotherapy, but current research results vary. Studies have also shown that sarcopenia is unrelated to irAEs (39). Our meta-analysis included eight clinical studies. The observed indicators include adverse reactions of major organs (interstitial pneumonia; colitis; hepatitis; thyroiditis; skin reactions; low levels of platelets and white blood cells; inflammation of the brain or spinal cord; neuromuscular adverse events, including myositis, Guillain-Barré syndrome, and myasthenia gravis; myocarditis and cardiac insufficiency; acute adrenal insufficiency; and nephritis), as well as adverse reactions of any grade (focusing on severe adverse reactions of grade 3 or 4). Compared with patients receiving traditional chemotherapy drugs, those receiving immune checkpoint blockers experience a lower incidence of adverse reactions. This meta-analysis treats all adverse reactions as a whole, but the results show that adverse reactions of any grade are not associated with sarcopenia.

Our meta-analysis strictly selected eligible studies and is the first to analyze the influence of sarcopenia on immuneinduced adverse reactions of any grade and severe grade. However, our meta-analysis had several limitations. First, the included studies were all retrospective in nature because 
of the lack of published randomized controlled studies, and there may have been selection bias in the enrolled patients. Second, the number of study samples in the enrolled study was relatively small, which is not sufficient to fully explain the research results. Third, although a subgroup analysis of severe irAEs was performed, the number of included studies was relatively small.

This study aimed to analyze the influence of sarcopenia on immune-induced adverse reactions and reveal the mechanism by which sarcopenia affects the outcomes of immunotherapy. The analysis results showed that sarcopenia did not increase the adverse reactions induced by ICIs. Therefore, patients with sarcopenia, especially those with advanced or poor physical conditions, can select immunotherapy, as indicated. Sarcopenia is not a contraindication to immunotherapy. However, due to the limited number of cases, more clinical trials are needed for further confirmation.

\section{Acknowledgments}

We would like to thank Editage (https://www.editage.cn) for English language editing.

Funding: This work was supported by Shenzhen Science and Technology Innovation Commission Project (JCYJ20190809100005672, ZDSYS20190902092855097, KCXFZ20200201101050887) and Shenzhen Sanming Project (SZSM201612041).

\section{Footnote}

Reporting Checklist: The authors have completed the PRISMA reporting checklist. Available at https://dx.doi. org/10.21037/tcr-21-1470

Conflicts of Interest: All authors have completed the ICMJE uniform disclosure form (available at https://dx.doi. org/10.21037/tcr-21-1470). The authors have no conflicts of interest to declare.

Ethical Statement: The authors are accountable for all aspects of the work in ensuring that questions related to the accuracy or integrity of any part of the work are appropriately investigated and resolved.

Open Access Statement: This is an Open Access article distributed in accordance with the Creative Commons Attribution-NonCommercial-NoDerivs 4.0 International
License (CC BY-NC-ND 4.0), which permits the noncommercial replication and distribution of the article with the strict proviso that no changes or edits are made and the original work is properly cited (including links to both the formal publication through the relevant DOI and the license). See: https://creativecommons.org/licenses/by-nc-nd/4.0/.

\section{References}

1. Doroshow DB, Sanmamed MF, Hastings K, et al. Immunotherapy in Non-Small Cell Lung Cancer: Facts and Hopes. Clin Cancer Res 2019;25:4592-602.

2. Wolchok JD, Hodi FS, Weber JS, et al. Development of ipilimumab: a novel immunotherapeutic approach for the treatment of advanced melanoma. Ann N Y Acad Sci 2013;1291:1-13.

3. Waterhouse D, Horn L, Reynolds C, et al. Safety profile of nivolumab administered as 30-min infusion: analysis of data from CheckMate 153. Cancer Chemother Pharmacol 2018;81:679-86.

4. Sun X, Roudi R, Dai T, et al. Immune-related adverse events associated with programmed cell death protein-1 and programmed cell death ligand 1 inhibitors for nonsmall cell lung cancer: a PRISMA systematic review and meta-analysis. BMC Cancer 2019;19:558.

5. Martin L, Birdsell L, Macdonald N, et al. Cancer cachexia in the age of obesity: skeletal muscle depletion is a powerful prognostic factor, independent of body mass index. J Clin Oncol 2013;31:1539-47.

6. Shachar SS, Williams GR, Muss HB, et al. Prognostic value of sarcopenia in adults with solid tumours: A meta-analysis and systematic review. Eur J Cancer 2016;57:58-67.

7. Nishioka N, Naito T, Notsu A, et al. Unfavorable impact of decreased muscle quality on the efficacy of immunotherapy for advanced non-small cell lung cancer. Cancer Med 2021;10:247-56.

8. Roch B, Coffy A, Jean-Baptiste S, et al. Cachexia - sarcopenia as a determinant of disease control rate and survival in non-small lung cancer patients receiving immune-checkpoint inhibitors. Lung Cancer 2020;143:19-26.

9. Adams SC, Segal RJ, McKenzie DC, et al. Impact of resistance and aerobic exercise on sarcopenia and dynapenia in breast cancer patients receiving adjuvant chemotherapy: a multicenter randomized controlled trial. Breast Cancer Res Treat 2016;158:497-507.

10. Bozzetti F. Forcing the vicious circle: sarcopenia increases toxicity, decreases response to chemotherapy and worsens 
with chemotherapy. Ann Oncol 2017;28:2107-18.

11. Heidelberger V, Goldwasser F, Kramkimel N, et al. Sarcopenic overweight is associated with early acute limiting toxicity of anti-PD1 checkpoint inhibitors in melanoma patients. Invest New Drugs 2017;35:436-41.

12. Li S, Wang T, Tong G, et al. Prognostic Impact of Sarcopenia on Clinical Outcomes in Malignancies Treated With Immune Checkpoint Inhibitors: A Systematic Review and Meta-Analysis. Front Oncol 2021;11:726257.

13. Cortellini A, Verna L, Porzio G, et al. Predictive value of skeletal muscle mass for immunotherapy with nivolumab in non-small cell lung cancer patients: A "hypothesis-generator" preliminary report. Thorac Cancer 2019;10:347-51.

14. Tsukagoshi M, Yokobori T, Yajima T, et al. Skeletal muscle mass predicts the outcome of nivolumab treatment for non-small cell lung cancer. Medicine (Baltimore) 2020;99:e19059.

15. Cortellini A, Bozzetti F, Palumbo P, et al. Weighing the role of skeletal muscle mass and muscle density in cancer patients receiving PD-1/PD-L1 checkpoint inhibitors: a multicenter real-life study. Sci Rep 2020;10:1456.

16. Hirsch L, Bellesoeur A, Boudou-Rouquette P, et al. The impact of body composition parameters on severe toxicity of nivolumab. Eur J Cancer 2020;124:170-7.

17. Shachar SS, Fried R, Shafran I, et al. Body composition as predictor of toxicity and outcomes in patients with metastatic non-small cell lung cancer (mNSCLC) receiving nivolumab (Nivo). J Clin Oncol 2018;36:e21010.

18. Kano M, Hihara J, Tokumoto N, et al. Association between skeletal muscle loss and the response to nivolumab immunotherapy in advanced gastric cancer patients. Int J Clin Oncol 2021;26:523-31.

19. Nascimento CM, Ingles M, Salvador-Pascual A, et al. Sarcopenia, frailty and their prevention by exercise. Free Radic Biol Med 2019;132:42-9.

20. Larsson L, Degens H, Li M, et al. Sarcopenia: AgingRelated Loss of Muscle Mass and Function. Physiol Rev 2019;99:427-511.

21. Doherty TJ. Invited review: Aging and sarcopenia. J Appl Physiol (1985) 2003;95:1717-27.

22. Marcell TJ. Sarcopenia: causes, consequences, and preventions. J Gerontol A Biol Sci Med Sci 2003;58:M911-6.

23. Beaudart C, Zaaria M, Pasleau F, et al. Health Outcomes of Sarcopenia: A Systematic Review and Meta-Analysis. PLoS One 2017;12:e0169548.

24. Farkona S, Diamandis EP, Blasutig IM. Cancer immunotherapy: the beginning of the end of cancer? BMC Med 2016;14:73.

25. Sharma P, Allison JP. Immune checkpoint targeting in cancer therapy: toward combination strategies with curative potential. Cell 2015;161:205-14.

26. Michot JM, Bigenwald C, Champiat S, et al. Immunerelated adverse events with immune checkpoint blockade: a comprehensive review. Eur J Cancer 2016;54:139-48.

27. Pardoll DM. The blockade of immune checkpoints in cancer immunotherapy. Nat Rev Cancer 2012;12:252-64.

28. Johansen A, Christensen SJ, Scheie D, et al. Neuromuscular adverse events associated with anti-PD-1 monoclonal antibodies: Systematic review. Neurology 2019;92:663-74.

29. Garg AD, Agostinis P. Cell death and immunity in cancer: From danger signals to mimicry of pathogen defense responses. Immunol Rev 2017;280:126-48.

30. Zhao Z, Zheng L, Chen W, et al. Delivery strategies of cancer immunotherapy: recent advances and future perspectives. J Hematol Oncol 2019;12:126.

31. Vega MC, Laviano A, Pimentel GD. Sarcopenia and chemotherapy-mediated toxicity. Einstein (Sao Paulo) 2016;14:580-4.

32. Wahlen BM, Mekkodathil A, Al-Thani H, et al. Impact of sarcopenia in trauma and surgical patient population: A literature review. Asian J Surg 2020;43:647-53.

33. Shachar SS, Deal AM, Weinberg M, et al. Body Composition as a Predictor of Toxicity in Patients Receiving Anthracycline and Taxane-Based Chemotherapy for Early-Stage Breast Cancer. Clin Cancer Res 2017;23:3537-43.

34. Chu MP, Li Y, Ghosh S, et al. Body composition is prognostic and predictive of ipilimumab activity in metastatic melanoma. J Cachexia Sarcopenia Muscle 2020;11:748-55.

35. Wang J, Cao L, Xu S. Sarcopenia affects clinical efficacy of immune checkpoint inhibitors in non-small cell lung cancer patients: A systematic review and meta-analysis. Int Immunopharmacol 2020;88:106907.

36. Nishioka N, Uchino J, Hirai S, et al. Association of Sarcopenia with and Efficacy of Anti-PD-1/PD-L1 Therapy in Non-Small-Cell Lung Cancer. J Clin Med 2019;8:450.

37. Loosen SH, van den Bosch V, Gorgulho J, et al. Progressive Sarcopenia Correlates with Poor Response and Outcome to Immune Checkpoint Inhibitor Therapy. J Clin Med 2021;10:1361.

38. Kim YY, Lee J, Jeong WK, et al. Prognostic significance of 
sarcopenia in microsatellite-stable gastric cancer patients treated with programmed death-1 inhibitors. Gastric Cancer 2021;24:457-66.

39. Young AC, Quach HT, Song H, et al. Impact of body

Cite this article as: Li S, Wang T, Lai W, Zhang M, Cheng B, Wang S, Tong G. Prognostic impact of sarcopenia on immune-related adverse events in malignancies received immune checkpoint inhibitors: a systematic review and metaanalysis. Transl Cancer Res 2021;10(12):5150-5158. doi: $10.21037 /$ tcr-21-1470 composition on outcomes from anti-PD1 +/- antiCTLA-4 treatment in melanoma. J Immunother Cancer 2020;8:e000821. 